\title{
Computational Study of the Reaction of Atomic Oxygen with Acetone in the Gas Phase
}

\author{
Hua Hou, Yuzhen Li, Baoshan Wang* \\ College of Chemistry and Molecular Sciences, Wuhan University, Wuhan, 430072, PR CHINA
}

[Submitted to J. Phys. Chem. A]

Table S1. The MEP data for the transition states TS1 and TS2 calculated at the MP2/6-311G(d,p) level of theory $\left(E_{M E P}^{M P 2 / 6-311 G(d, p)}\right)$ and interpolated at the FCC/CBS level $\left(V_{a}^{G}\right)$. The reaction coordinates (s) are in $a m u^{1 / 2}$ bohr. All energies are in $\mathrm{kcal} / \mathrm{mol}$. 
Table S1. The MEP data for the transition states TS1 and TS2 calculated at the MP2/6-311G(d,p) level of theory $\left(E_{M E P}^{M P 2 / 6-311 G(d, p)}\right)$ and interpolated at the FCC/CBS level $\left(V_{a}^{G}\right)$. The reaction coordinates (s) are in amu ${ }^{1 / 2}$ bohr. All energies are in $\mathrm{kcal} / \mathrm{mol}$.

\begin{tabular}{|c|c|c|c|c|c|}
\hline \multicolumn{3}{|c|}{ TS1 } & \multicolumn{3}{|c|}{ TS2 } \\
\hline$s$ & $E_{M E P}^{M P 2 / 6-311 G(d, p)}$ & $V_{a}^{G}$ & $s$ & $E_{M E P}^{M P 2 / 6-311 G(d, p)}$ & $V_{a}^{G}$ \\
\hline-3.13 & 2.002 & 0.472 & -4 & 2.372 & 0.382 \\
\hline-3.12 & 2.027 & 0.487 & -3.99 & 2.391 & 0.379 \\
\hline-3.11 & 2.046 & 0.495 & -3.98 & 2.416 & 0.383 \\
\hline-3.1 & 2.065 & 0.503 & -3.97 & 2.441 & 0.386 \\
\hline-3.09 & 2.09 & 0.518 & -3.96 & 2.466 & 0.389 \\
\hline-3.08 & 2.108 & 0.526 & -3.95 & 2.485 & 0.386 \\
\hline-3.07 & 2.134 & 0.54 & -3.94 & 2.51 & 0.389 \\
\hline-3.06 & 2.152 & 0.548 & -3.93 & 2.535 & 0.392 \\
\hline-3.05 & 2.177 & 0.562 & -3.92 & 2.56 & 0.395 \\
\hline-3.04 & 2.203 & 0.576 & -3.91 & 2.579 & 0.391 \\
\hline-3.03 & 2.221 & 0.584 & -3.9 & 2.604 & 0.394 \\
\hline-3.02 & 2.246 & 0.598 & -3.89 & 2.629 & 0.396 \\
\hline-3.01 & 2.265 & 0.605 & -3.88 & 2.654 & 0.398 \\
\hline-3 & 2.29 & 0.619 & -3.87 & 2.679 & 0.401 \\
\hline-2.99 & 2.316 & 0.633 & -3.86 & 2.705 & 0.403 \\
\hline-2.98 & 2.334 & 0.64 & -3.85 & 2.73 & 0.405 \\
\hline-2.97 & 2.359 & 0.654 & -3.84 & 2.748 & 0.4 \\
\hline-2.96 & 2.385 & 0.667 & -3.83 & 2.774 & 0.402 \\
\hline-2.95 & 2.41 & 0.681 & -3.82 & 2.799 & 0.403 \\
\hline-2.94 & 2.428 & 0.688 & -3.81 & 2.824 & 0.405 \\
\hline-2.93 & 2.454 & 0.701 & -3.8 & 2.849 & 0.406 \\
\hline-2.92 & 2.479 & 0.714 & -3.79 & 2.874 & 0.407 \\
\hline-2.91 & 2.504 & 0.727 & -3.78 & 2.905 & 0.415 \\
\hline-2.9 & 2.529 & 0.74 & -3.77 & 2.93 & 0.416 \\
\hline-2.89 & 2.554 & 0.753 & -3.76 & 2.956 & 0.416 \\
\hline-2.88 & 2.579 & 0.766 & -3.75 & 2.981 & 0.417 \\
\hline-2.87 & 2.604 & 0.779 & -3.74 & 3.006 & 0.418 \\
\hline-2.86 & 2.629 & 0.791 & -3.73 & 3.031 & 0.418 \\
\hline-2.85 & 2.654 & 0.804 & -3.72 & 3.056 & 0.418 \\
\hline-2.84 & 2.679 & 0.816 & -3.71 & 3.087 & 0.425 \\
\hline-2.83 & 2.705 & 0.829 & -3.7 & 3.112 & 0.425 \\
\hline-2.82 & 2.73 & 0.841 & -3.69 & 3.138 & 0.425 \\
\hline-2.81 & 2.755 & 0.854 & -3.68 & 3.163 & 0.425 \\
\hline-2.8 & 2.78 & 0.866 & -3.67 & 3.194 & 0.431 \\
\hline-2.79 & 2.805 & 0.878 & -3.66 & 3.219 & 0.43 \\
\hline-2.78 & 2.83 & 0.89 & -3.65 & 3.244 & 0.43 \\
\hline
\end{tabular}




\begin{tabular}{|c|c|c|c|c|c|}
\hline-2.77 & 2.861 & 0.908 & -3.64 & 3.276 & 0.436 \\
\hline-2.76 & 2.887 & 0.92 & -3.63 & 3.301 & 0.435 \\
\hline-2.75 & 2.912 & 0.932 & -3.62 & 3.326 & 0.434 \\
\hline-2.74 & 2.937 & 0.943 & -3.61 & 3.357 & 0.439 \\
\hline-2.73 & 2.968 & 0.961 & -3.6 & 3.382 & 0.438 \\
\hline-2.72 & 2.993 & 0.973 & -3.59 & 3.414 & 0.443 \\
\hline-2.71 & 3.018 & 0.984 & -3.58 & 3.439 & 0.442 \\
\hline-2.7 & 3.05 & 1.002 & -3.57 & 3.47 & 0.447 \\
\hline-2.69 & 3.075 & 1.013 & -3.56 & 3.495 & 0.445 \\
\hline-2.68 & 3.1 & 1.024 & -3.55 & 3.527 & 0.45 \\
\hline-2.67 & 3.131 & 1.041 & -3.54 & 3.558 & 0.454 \\
\hline-2.66 & 3.156 & 1.052 & -3.53 & 3.583 & 0.452 \\
\hline-2.65 & 3.188 & 1.069 & -3.52 & 3.614 & 0.456 \\
\hline-2.64 & 3.213 & 1.08 & -3.51 & 3.646 & 0.461 \\
\hline-2.63 & 3.244 & 1.097 & -3.5 & 3.671 & 0.458 \\
\hline-2.62 & 3.276 & 1.114 & -3.49 & 3.702 & 0.462 \\
\hline-2.61 & 3.301 & 1.124 & -3.48 & 3.734 & 0.466 \\
\hline-2.6 & 3.332 & 1.141 & -3.47 & 3.759 & 0.463 \\
\hline-2.59 & 3.363 & 1.158 & -3.46 & 3.79 & 0.467 \\
\hline-2.58 & 3.389 & 1.168 & -3.45 & 3.822 & 0.47 \\
\hline-2.57 & 3.42 & 1.184 & -3.44 & 3.853 & 0.474 \\
\hline-2.56 & 3.451 & 1.201 & -3.43 & 3.884 & 0.477 \\
\hline-2.55 & 3.476 & 1.211 & -3.42 & 3.916 & 0.48 \\
\hline-2.54 & 3.508 & 1.227 & -3.41 & 3.947 & 0.483 \\
\hline-2.53 & 3.539 & 1.243 & -3.4 & 3.972 & 0.479 \\
\hline-2.52 & 3.571 & 1.259 & -3.39 & 4.004 & 0.482 \\
\hline-2.51 & 3.602 & 1.275 & -3.38 & 4.035 & 0.484 \\
\hline-2.5 & 3.633 & 1.29 & -3.37 & 4.066 & 0.487 \\
\hline-2.49 & 3.665 & 1.306 & -3.36 & 4.098 & 0.489 \\
\hline-2.48 & 3.696 & 1.321 & -3.35 & 4.135 & 0.498 \\
\hline-2.47 & 3.727 & 1.337 & -3.34 & 4.167 & 0.5 \\
\hline-2.46 & 3.759 & 1.352 & -3.33 & 4.198 & 0.502 \\
\hline-2.45 & 3.79 & 1.367 & -3.32 & 4.229 & 0.504 \\
\hline-2.44 & 3.822 & 1.383 & -3.31 & 4.261 & 0.506 \\
\hline-2.43 & 3.853 & 1.398 & -3.3 & 4.292 & 0.508 \\
\hline-2.42 & 3.884 & 1.413 & -3.29 & 4.324 & 0.509 \\
\hline-2.41 & 3.916 & 1.427 & -3.28 & 4.361 & 0.517 \\
\hline-2.4 & 3.953 & 1.448 & -3.27 & 4.393 & 0.519 \\
\hline-2.39 & 3.985 & 1.463 & -3.26 & 4.424 & 0.52 \\
\hline-2.38 & 4.016 & 1.478 & -3.25 & 4.462 & 0.527 \\
\hline-2.37 & 4.047 & 1.492 & -3.24 & 4.493 & 0.528 \\
\hline-2.36 & 4.085 & 1.513 & -3.23 & 4.524 & 0.529 \\
\hline-2.35 & 4.116 & 1.527 & -3.22 & 4.562 & 0.537 \\
\hline-2.34 & 4.154 & 1.547 & -3.21 & 4.593 & 0.537 \\
\hline
\end{tabular}




\begin{tabular}{|c|c|c|c|c|c|}
\hline-2.33 & 4.185 & 1.561 & -3.2 & 4.631 & 0.544 \\
\hline-2.32 & 4.217 & 1.575 & -3.19 & 4.662 & 0.545 \\
\hline-2.31 & 4.255 & 1.595 & -3.18 & 4.7 & 0.551 \\
\hline-2.3 & 4.286 & 1.609 & -3.17 & 4.731 & 0.552 \\
\hline-2.29 & 4.324 & 1.629 & -3.16 & 4.769 & 0.558 \\
\hline-2.28 & 4.361 & 1.648 & -3.15 & 4.807 & 0.565 \\
\hline-2.27 & 4.393 & 1.662 & -3.14 & 4.838 & 0.565 \\
\hline-2.26 & 4.43 & 1.681 & -3.13 & 4.876 & 0.571 \\
\hline-2.25 & 4.468 & 1.701 & -3.12 & 4.913 & 0.577 \\
\hline-2.24 & 4.499 & 1.714 & -3.11 & 4.945 & 0.577 \\
\hline-2.23 & 4.537 & 1.733 & -3.1 & 4.982 & 0.582 \\
\hline-2.22 & 4.575 & 1.752 & -3.09 & 5.02 & 0.588 \\
\hline-2.21 & 4.612 & 1.771 & -3.08 & 5.058 & 0.594 \\
\hline-2.2 & 4.65 & 1.79 & -3.07 & 5.089 & 0.593 \\
\hline-2.19 & 4.681 & 1.803 & -3.06 & 5.127 & 0.599 \\
\hline-2.18 & 4.719 & 1.821 & -3.05 & 5.164 & 0.604 \\
\hline-2.17 & 4.757 & 1.84 & -3.04 & 5.202 & 0.609 \\
\hline-2.16 & 4.794 & 1.858 & -3.03 & 5.24 & 0.614 \\
\hline-2.15 & 4.832 & 1.877 & -3.02 & 5.277 & 0.62 \\
\hline-2.14 & 4.869 & 1.895 & -3.01 & 5.315 & 0.624 \\
\hline-2.13 & 4.907 & 1.913 & -3 & 5.353 & 0.629 \\
\hline-2.12 & 4.951 & 1.937 & -2.99 & 5.39 & 0.634 \\
\hline-2.11 & 4.989 & 1.955 & -2.98 & 5.428 & 0.639 \\
\hline-2.1 & 5.026 & 1.973 & -2.97 & 5.472 & 0.65 \\
\hline-2.09 & 5.064 & 1.991 & -2.96 & 5.51 & 0.654 \\
\hline-2.08 & 5.108 & 2.015 & -2.95 & 5.547 & 0.659 \\
\hline-2.07 & 5.146 & 2.032 & -2.94 & 5.585 & 0.663 \\
\hline-2.06 & 5.183 & 2.049 & -2.93 & 5.629 & 0.674 \\
\hline-2.05 & 5.227 & 2.073 & -2.92 & 5.666 & 0.678 \\
\hline-2.04 & 5.265 & 2.09 & -2.91 & 5.704 & 0.682 \\
\hline-2.03 & 5.302 & 2.107 & -2.9 & 5.748 & 0.692 \\
\hline-2.02 & 5.346 & 2.13 & -2.89 & 5.786 & 0.696 \\
\hline-2.01 & 5.384 & 2.147 & -2.88 & 5.823 & 0.699 \\
\hline-2 & 5.428 & 2.17 & -2.87 & 5.867 & 0.709 \\
\hline-1.99 & 5.472 & 2.193 & -2.86 & 5.905 & 0.713 \\
\hline-1.98 & 5.51 & 2.209 & -2.85 & 5.949 & 0.723 \\
\hline-1.97 & 5.553 & 2.232 & -2.84 & 5.993 & 0.733 \\
\hline-1.96 & 5.597 & 2.254 & -2.83 & 6.03 & 0.736 \\
\hline-1.95 & 5.635 & 2.27 & -2.82 & 6.074 & 0.745 \\
\hline-1.94 & 5.679 & 2.293 & -2.81 & 6.118 & 0.755 \\
\hline-1.93 & 5.723 & 2.315 & -2.8 & 6.156 & 0.758 \\
\hline-1.92 & 5.767 & 2.337 & -2.79 & 6.2 & 0.767 \\
\hline-1.91 & 5.811 & 2.359 & -2.78 & 6.244 & 0.776 \\
\hline-1.9 & 5.855 & 2.381 & -2.77 & 6.288 & 0.785 \\
\hline
\end{tabular}




\begin{tabular}{|c|c|c|c|c|c|}
\hline-1.89 & 5.899 & 2.402 & -2.76 & 6.325 & 0.788 \\
\hline-1.88 & 5.943 & 2.424 & -2.75 & 6.369 & 0.797 \\
\hline-1.87 & 5.986 & 2.445 & -2.74 & 6.413 & 0.806 \\
\hline-1.86 & 6.03 & 2.467 & -2.73 & 6.457 & 0.815 \\
\hline-1.85 & 6.074 & 2.488 & -2.72 & 6.501 & 0.823 \\
\hline-1.84 & 6.118 & 2.509 & -2.71 & 6.545 & 0.832 \\
\hline-1.83 & 6.168 & 2.536 & -2.7 & 6.589 & 0.84 \\
\hline-1.82 & 6.212 & 2.557 & -2.69 & 6.633 & 0.849 \\
\hline-1.81 & 6.256 & 2.578 & -2.68 & 6.677 & 0.857 \\
\hline-1.8 & 6.306 & 2.605 & -2.67 & 6.727 & 0.872 \\
\hline-1.79 & 6.35 & 2.625 & -2.66 & 6.771 & 0.88 \\
\hline-1.78 & 6.394 & 2.646 & -2.65 & 6.815 & 0.888 \\
\hline-1.77 & 6.445 & 2.672 & -2.64 & 6.859 & 0.896 \\
\hline-1.76 & 6.488 & 2.693 & -2.63 & 6.909 & 0.911 \\
\hline-1.75 & 6.539 & 2.719 & -2.62 & 6.953 & 0.918 \\
\hline-1.74 & 6.589 & 2.745 & -2.61 & 6.997 & 0.926 \\
\hline-1.73 & 6.633 & 2.765 & -2.6 & 7.047 & 0.94 \\
\hline-1.72 & 6.683 & 2.791 & -2.59 & 7.091 & 0.948 \\
\hline-1.71 & 6.733 & 2.817 & -2.58 & 7.141 & 0.962 \\
\hline-1.7 & 6.777 & 2.837 & -2.57 & 7.185 & 0.97 \\
\hline-1.69 & 6.827 & 2.862 & -2.56 & 7.235 & 0.984 \\
\hline-1.68 & 6.878 & 2.888 & -2.55 & 7.285 & 0.997 \\
\hline-1.67 & 6.928 & 2.913 & -2.54 & 7.329 & 1.005 \\
\hline-1.66 & 6.978 & 2.939 & -2.53 & 7.38 & 1.018 \\
\hline-1.65 & 7.028 & 2.964 & -2.52 & 7.43 & 1.032 \\
\hline-1.64 & 7.078 & 2.989 & -2.51 & 7.474 & 1.039 \\
\hline-1.63 & 7.129 & 3.014 & -2.5 & 7.524 & 1.052 \\
\hline-1.62 & 7.179 & 3.039 & -2.49 & 7.574 & 1.066 \\
\hline-1.61 & 7.229 & 3.064 & -2.48 & 7.624 & 1.079 \\
\hline-1.6 & 7.285 & 3.095 & -2.47 & 7.674 & 1.092 \\
\hline-1.59 & 7.336 & 3.12 & -2.46 & 7.725 & 1.106 \\
\hline-1.58 & 7.386 & 3.144 & -2.45 & 7.775 & 1.119 \\
\hline-1.57 & 7.436 & 3.169 & -2.44 & 7.825 & 1.132 \\
\hline-1.56 & 7.492 & 3.199 & -2.43 & 7.875 & 1.145 \\
\hline-1.55 & 7.543 & 3.223 & -2.42 & 7.925 & 1.158 \\
\hline-1.54 & 7.599 & 3.254 & -2.41 & 7.976 & 1.17 \\
\hline-1.53 & 7.649 & 3.278 & -2.4 & 8.026 & 1.183 \\
\hline-1.52 & 7.706 & 3.308 & -2.39 & 8.082 & 1.203 \\
\hline-1.51 & 7.756 & 3.332 & -2.38 & 8.133 & 1.215 \\
\hline-1.5 & 7.812 & 3.361 & -2.37 & 8.183 & 1.228 \\
\hline-1.49 & 7.869 & 3.391 & -2.36 & 8.239 & 1.247 \\
\hline-1.48 & 7.925 & 3.421 & -2.35 & 8.289 & 1.259 \\
\hline-1.47 & 7.976 & 3.444 & -2.34 & 8.34 & 1.272 \\
\hline-1.46 & 8.032 & 3.474 & -2.33 & 8.396 & 1.291 \\
\hline
\end{tabular}




\begin{tabular}{|c|c|c|c|c|c|}
\hline-1.45 & 8.089 & 3.503 & -2.32 & 8.446 & 1.303 \\
\hline-1.44 & 8.145 & 3.533 & -2.31 & 8.503 & 1.322 \\
\hline-1.43 & 8.202 & 3.562 & -2.3 & 8.559 & 1.341 \\
\hline-1.42 & 8.258 & 3.591 & -2.29 & 8.609 & 1.353 \\
\hline-1.41 & 8.315 & 3.62 & -2.28 & 8.666 & 1.371 \\
\hline-1.4 & 8.371 & 3.649 & -2.27 & 8.722 & 1.39 \\
\hline-1.39 & 8.427 & 3.678 & -2.26 & 8.779 & 1.409 \\
\hline-1.38 & 8.49 & 3.713 & -2.25 & 8.829 & 1.421 \\
\hline-1.37 & 8.547 & 3.742 & -2.24 & 8.886 & 1.439 \\
\hline-1.36 & 8.603 & 3.771 & -2.23 & 8.942 & 1.457 \\
\hline-1.35 & 8.66 & 3.799 & -2.22 & 8.998 & 1.476 \\
\hline-1.34 & 8.722 & 3.834 & -2.21 & 9.055 & 1.494 \\
\hline-1.33 & 8.779 & 3.862 & -2.2 & 9.111 & 1.512 \\
\hline-1.32 & 8.842 & 3.897 & -2.19 & 9.168 & 1.531 \\
\hline-1.31 & 8.898 & 3.925 & -2.18 & 9.224 & 1.549 \\
\hline-1.3 & 8.961 & 3.959 & -2.17 & 9.281 & 1.567 \\
\hline-1.29 & 9.017 & 3.987 & -2.16 & 9.344 & 1.592 \\
\hline-1.28 & 9.08 & 4.021 & -2.15 & 9.4 & 1.61 \\
\hline-1.27 & 9.143 & 4.056 & -2.14 & 9.457 & 1.628 \\
\hline-1.26 & 9.206 & 4.09 & -2.13 & 9.513 & 1.646 \\
\hline-1.25 & 9.262 & 4.117 & -2.12 & 9.576 & 1.67 \\
\hline-1.24 & 9.325 & 4.151 & -2.11 & 9.632 & 1.688 \\
\hline-1.23 & 9.388 & 4.185 & -2.1 & 9.695 & 1.713 \\
\hline-1.22 & 9.45 & 4.219 & -2.09 & 9.751 & 1.73 \\
\hline-1.21 & 9.513 & 4.252 & -2.08 & 9.814 & 1.755 \\
\hline-1.2 & 9.576 & 4.286 & -2.07 & 9.871 & 1.773 \\
\hline-1.19 & 9.639 & 4.32 & -2.06 & 9.933 & 1.797 \\
\hline-1.18 & 9.701 & 4.353 & -2.05 & 9.996 & 1.821 \\
\hline-1.17 & 9.77 & 4.393 & -2.04 & 10.053 & 1.839 \\
\hline-1.16 & 9.833 & 4.426 & -2.03 & 10.115 & 1.863 \\
\hline-1.15 & 9.896 & 4.459 & -2.02 & 10.178 & 1.887 \\
\hline-1.14 & 9.959 & 4.493 & -2.01 & 10.241 & 1.912 \\
\hline-1.13 & 10.028 & 4.532 & -2 & 10.304 & 1.936 \\
\hline-1.12 & 10.09 & 4.565 & -1.99 & 10.366 & 1.96 \\
\hline-1.11 & 10.159 & 4.604 & -1.98 & 10.429 & 1.984 \\
\hline-1.1 & 10.222 & 4.637 & -1.97 & 10.492 & 2.008 \\
\hline-1.09 & 10.291 & 4.677 & -1.96 & 10.555 & 2.032 \\
\hline-1.08 & 10.354 & 4.71 & -1.95 & 10.617 & 2.056 \\
\hline-1.07 & 10.423 & 4.749 & -1.94 & 10.68 & 2.08 \\
\hline-1.06 & 10.492 & 4.788 & -1.93 & 10.743 & 2.104 \\
\hline-1.05 & 10.555 & 4.82 & -1.92 & 10.812 & 2.135 \\
\hline-1.04 & 10.624 & 4.859 & -1.91 & 10.875 & 2.159 \\
\hline-1.03 & 10.693 & 4.898 & -1.9 & 10.937 & 2.183 \\
\hline-1.02 & 10.762 & 4.937 & -1.89 & 11.007 & 2.214 \\
\hline
\end{tabular}




\begin{tabular}{|c|c|c|c|c|c|}
\hline-1.01 & 10.831 & 4.976 & -1.88 & 11.069 & 2.238 \\
\hline-1 & 10.9 & 5.015 & -1.87 & 11.138 & 2.268 \\
\hline-0.99 & 10.969 & 5.054 & -1.86 & 11.201 & 2.292 \\
\hline-0.98 & 11.038 & 5.093 & -1.85 & 11.27 & 2.323 \\
\hline-0.97 & 11.107 & 5.131 & -1.84 & 11.333 & 2.347 \\
\hline-0.96 & 11.176 & 5.17 & -1.83 & 11.402 & 2.377 \\
\hline-0.95 & 11.245 & 5.209 & -1.82 & 11.471 & 2.408 \\
\hline-0.94 & 11.32 & 5.254 & -1.81 & 11.534 & 2.432 \\
\hline-0.93 & 11.389 & 5.292 & -1.8 & 11.603 & 2.462 \\
\hline-0.92 & 11.458 & 5.331 & -1.79 & 11.672 & 2.493 \\
\hline-0.91 & 11.534 & 5.376 & -1.78 & 11.741 & 2.523 \\
\hline-0.9 & 11.603 & 5.414 & -1.77 & 11.81 & 2.554 \\
\hline-0.89 & 11.678 & 5.459 & -1.76 & 11.879 & 2.585 \\
\hline-0.88 & 11.747 & 5.498 & -1.75 & 11.948 & 2.615 \\
\hline-0.87 & 11.822 & 5.542 & -1.74 & 12.017 & 2.646 \\
\hline-0.86 & 11.891 & 5.581 & -1.73 & 12.086 & 2.676 \\
\hline-0.85 & 11.967 & 5.626 & -1.72 & 12.155 & 2.707 \\
\hline-0.84 & 12.042 & 5.671 & -1.71 & 12.224 & 2.737 \\
\hline-0.83 & 12.111 & 5.709 & -1.7 & 12.293 & 2.768 \\
\hline-0.82 & 12.186 & 5.754 & -1.69 & 12.368 & 2.805 \\
\hline-0.81 & 12.262 & 5.799 & -1.68 & 12.437 & 2.836 \\
\hline-0.8 & 12.337 & 5.843 & -1.67 & 12.506 & 2.867 \\
\hline-0.79 & 12.412 & 5.888 & -1.66 & 12.582 & 2.904 \\
\hline-0.78 & 12.487 & 5.933 & -1.65 & 12.651 & 2.935 \\
\hline-0.77 & 12.563 & 5.978 & -1.64 & 12.726 & 2.972 \\
\hline-0.76 & 12.638 & 6.023 & -1.63 & 12.795 & 3.003 \\
\hline-0.75 & 12.713 & 6.068 & -1.62 & 12.87 & 3.04 \\
\hline-0.74 & 12.789 & 6.113 & -1.61 & 12.946 & 3.077 \\
\hline-0.73 & 12.864 & 6.158 & -1.6 & 13.015 & 3.108 \\
\hline-0.72 & 12.946 & 6.209 & -1.59 & 13.09 & 3.146 \\
\hline-0.71 & 13.021 & 6.254 & -1.58 & 13.165 & 3.183 \\
\hline-0.7 & 13.096 & 6.299 & -1.57 & 13.234 & 3.214 \\
\hline-0.69 & 13.178 & 6.35 & -1.56 & 13.309 & 3.252 \\
\hline-0.68 & 13.253 & 6.395 & -1.55 & 13.385 & 3.289 \\
\hline-0.67 & 13.328 & 6.44 & -1.54 & 13.46 & 3.327 \\
\hline-0.66 & 13.41 & 6.492 & -1.53 & 13.535 & 3.365 \\
\hline-0.65 & 13.485 & 6.537 & -1.52 & 13.611 & 3.402 \\
\hline-0.64 & 13.567 & 6.588 & -1.51 & 13.686 & 3.44 \\
\hline-0.63 & 13.648 & 6.64 & -1.5 & 13.761 & 3.478 \\
\hline-0.62 & 13.724 & 6.685 & -1.49 & 13.837 & 3.516 \\
\hline-0.61 & 13.805 & 6.737 & -1.48 & 13.912 & 3.553 \\
\hline-0.6 & 13.887 & 6.789 & -1.47 & 13.993 & 3.598 \\
\hline-0.59 & 13.962 & 6.834 & -1.46 & 14.069 & 3.636 \\
\hline-0.58 & 14.044 & 6.886 & -1.45 & 14.144 & 3.674 \\
\hline
\end{tabular}




\begin{tabular}{|c|c|c|c|c|c|}
\hline-0.57 & 14.125 & 6.938 & -1.44 & 14.226 & 3.719 \\
\hline-0.56 & 14.207 & 6.99 & -1.43 & 14.301 & 3.757 \\
\hline-0.55 & 14.288 & 7.042 & -1.42 & 14.376 & 3.795 \\
\hline-0.54 & 14.37 & 7.094 & -1.41 & 14.458 & 3.84 \\
\hline-0.53 & 14.452 & 7.146 & -1.4 & 14.533 & 3.878 \\
\hline-0.52 & 14.533 & 7.199 & -1.39 & 14.615 & 3.923 \\
\hline-0.51 & 14.615 & 7.251 & -1.38 & 14.69 & 3.961 \\
\hline-0.5 & 14.703 & 7.31 & -1.37 & 14.772 & 4.006 \\
\hline-0.49 & 14.784 & 7.362 & -1.36 & 14.853 & 4.051 \\
\hline-0.48 & 14.866 & 7.415 & -1.35 & 14.928 & 4.09 \\
\hline-0.47 & 14.954 & 7.474 & -1.34 & 15.01 & 4.135 \\
\hline-0.46 & 15.035 & 7.526 & -1.33 & 15.092 & 4.18 \\
\hline-0.45 & 15.123 & 7.586 & -1.32 & 15.167 & 4.219 \\
\hline-0.44 & 15.211 & 7.645 & -1.31 & 15.248 & 4.265 \\
\hline-0.43 & 15.292 & 7.698 & -1.3 & 15.33 & 4.31 \\
\hline-0.42 & 15.387 & 7.764 & -1.29 & 15.412 & 4.356 \\
\hline-0.41 & 15.474 & 7.823 & -1.28 & 15.493 & 4.401 \\
\hline-0.4 & 15.569 & 7.889 & -1.27 & 15.575 & 4.447 \\
\hline-0.39 & 15.663 & 7.955 & -1.26 & 15.656 & 4.493 \\
\hline-0.38 & 15.757 & 8.021 & -1.25 & 15.738 & 4.539 \\
\hline-0.37 & 15.857 & 8.094 & -1.24 & 15.82 & 4.585 \\
\hline-0.36 & 15.964 & 8.173 & -1.23 & 15.901 & 4.631 \\
\hline-0.35 & 16.077 & 8.258 & -1.22 & 15.983 & 4.677 \\
\hline-0.34 & 16.19 & 8.343 & -1.21 & 16.064 & 4.723 \\
\hline-0.33 & 16.309 & 8.435 & -1.2 & 16.146 & 4.769 \\
\hline-0.32 & 16.434 & 8.533 & -1.19 & 16.234 & 4.822 \\
\hline-0.31 & 16.566 & 8.638 & -1.18 & 16.315 & 4.869 \\
\hline-0.3 & 16.698 & 8.743 & -1.17 & 16.397 & 4.915 \\
\hline-0.29 & 16.842 & 8.86 & -1.16 & 16.478 & 4.962 \\
\hline-0.28 & 16.987 & 8.978 & -1.15 & 16.566 & 5.015 \\
\hline-0.27 & 17.131 & 9.095 & -1.14 & 16.648 & 5.062 \\
\hline-0.26 & 17.282 & 9.22 & -1.13 & 16.729 & 5.109 \\
\hline-0.25 & 17.438 & 9.35 & -1.12 & 16.817 & 5.163 \\
\hline-0.24 & 17.595 & 9.481 & -1.11 & 16.899 & 5.21 \\
\hline-0.23 & 17.752 & 9.612 & -1.1 & 16.987 & 5.264 \\
\hline-0.22 & 17.909 & 9.743 & -1.09 & 17.068 & 5.311 \\
\hline-0.21 & 18.066 & 9.874 & -1.08 & 17.156 & 5.365 \\
\hline-0.2 & 18.223 & 10.005 & -1.07 & 17.238 & 5.413 \\
\hline-0.19 & 18.38 & 10.136 & -1.06 & 17.326 & 5.467 \\
\hline-0.18 & 18.53 & 10.262 & -1.05 & 17.407 & 5.515 \\
\hline-0.17 & 18.681 & 10.387 & -1.04 & 17.495 & 5.569 \\
\hline-0.16 & 18.825 & 10.506 & -1.03 & 17.577 & 5.617 \\
\hline-0.15 & 18.97 & 10.626 & -1.02 & 17.664 & 5.672 \\
\hline-0.14 & 19.101 & 10.733 & -1.01 & 17.752 & 5.727 \\
\hline
\end{tabular}




\begin{tabular}{|c|c|c|c|c|c|}
\hline-0.13 & 19.233 & 10.84 & -1 & 17.834 & 5.775 \\
\hline-0.12 & 19.352 & 10.935 & -0.99 & 17.922 & 5.83 \\
\hline-0.11 & 19.465 & 11.024 & -0.98 & 18.01 & 5.885 \\
\hline-0.1 & 19.572 & 11.107 & -0.97 & 18.091 & 5.934 \\
\hline-0.09 & 19.672 & 11.183 & -0.96 & 18.179 & 5.989 \\
\hline-0.08 & 19.76 & 11.247 & -0.95 & 18.267 & 6.045 \\
\hline-0.07 & 19.842 & 11.306 & -0.94 & 18.355 & 6.1 \\
\hline-0.06 & 19.911 & 11.351 & -0.93 & 18.436 & 6.15 \\
\hline-0.05 & 19.974 & 11.391 & -0.92 & 18.524 & 6.206 \\
\hline-0.04 & 20.018 & 11.412 & -0.91 & 18.612 & 6.262 \\
\hline-0.03 & 20.055 & 11.427 & -0.9 & 18.7 & 6.318 \\
\hline-0.02 & 20.087 & 11.436 & -0.89 & 18.781 & 6.368 \\
\hline-0.01 & 20.105 & 11.432 & -0.88 & 18.869 & 6.424 \\
\hline 0 & 20.105 & 11.41 & -0.87 & 18.957 & 6.481 \\
\hline 0.01 & 20.099 & 11.382 & -0.86 & 19.045 & 6.537 \\
\hline 0.02 & 20.087 & 11.347 & -0.85 & 19.133 & 6.594 \\
\hline 0.03 & 20.061 & 11.301 & -0.84 & 19.214 & 6.645 \\
\hline 0.04 & 20.024 & 11.242 & -0.83 & 19.302 & 6.702 \\
\hline 0.05 & 19.974 & 11.17 & -0.82 & 19.39 & 6.759 \\
\hline 0.06 & 19.917 & 11.093 & -0.81 & 19.478 & 6.816 \\
\hline 0.07 & 19.848 & 11.003 & -0.8 & 19.566 & 6.874 \\
\hline 0.08 & 19.773 & 10.907 & -0.79 & 19.647 & 6.925 \\
\hline 0.09 & 19.691 & 10.805 & -0.78 & 19.735 & 6.983 \\
\hline 0.1 & 19.603 & 10.696 & -0.77 & 19.823 & 7.041 \\
\hline 0.11 & 19.503 & 10.576 & -0.76 & 19.911 & 7.099 \\
\hline 0.12 & 19.396 & 10.449 & -0.75 & 19.992 & 7.151 \\
\hline 0.13 & 19.29 & 10.323 & -0.74 & 20.08 & 7.21 \\
\hline 0.14 & 19.17 & 10.184 & -0.73 & 20.168 & 7.268 \\
\hline 0.15 & 19.051 & 10.045 & -0.72 & 20.25 & 7.32 \\
\hline 0.16 & 18.926 & 9.901 & -0.71 & 20.338 & 7.379 \\
\hline 0.17 & 18.794 & 9.75 & -0.7 & 20.425 & 7.439 \\
\hline 0.18 & 18.662 & 9.599 & -0.69 & 20.507 & 7.491 \\
\hline 0.19 & 18.53 & 9.449 & -0.68 & 20.595 & 7.551 \\
\hline 0.2 & 18.392 & 9.292 & -0.67 & 20.683 & 7.611 \\
\hline 0.21 & 18.261 & 9.142 & -0.66 & 20.764 & 7.664 \\
\hline 0.22 & 18.122 & 8.986 & -0.65 & 20.852 & 7.724 \\
\hline 0.23 & 17.991 & 8.836 & -0.64 & 20.934 & 7.777 \\
\hline 0.24 & 17.853 & 8.68 & -0.63 & 21.015 & 7.831 \\
\hline 0.25 & 17.721 & 8.531 & -0.62 & 21.103 & 7.892 \\
\hline 0.26 & 17.595 & 8.388 & -0.61 & 21.185 & 7.946 \\
\hline 0.27 & 17.47 & 8.245 & -0.6 & 21.273 & 8.007 \\
\hline 0.28 & 17.344 & 8.102 & -0.59 & 21.354 & 8.061 \\
\hline 0.29 & 17.225 & 7.966 & -0.58 & 21.436 & 8.116 \\
\hline 0.3 & 17.112 & 7.836 & -0.57 & 21.517 & 8.17 \\
\hline
\end{tabular}




\begin{tabular}{|c|c|c|c|c|c|}
\hline 0.31 & 17.006 & 7.713 & -0.56 & 21.599 & 8.225 \\
\hline 0.32 & 16.899 & 7.59 & -0.55 & 21.68 & 8.281 \\
\hline 0.33 & 16.798 & 7.473 & -0.54 & 21.762 & 8.336 \\
\hline 0.34 & 16.704 & 7.363 & -0.53 & 21.844 & 8.392 \\
\hline 0.35 & 16.61 & 7.253 & -0.52 & 21.925 & 8.447 \\
\hline 0.36 & 16.522 & 7.149 & -0.51 & 22.007 & 8.503 \\
\hline 0.37 & 16.434 & 7.046 & -0.5 & 22.088 & 8.559 \\
\hline 0.38 & 16.353 & 6.949 & -0.49 & 22.164 & 8.609 \\
\hline 0.39 & 16.271 & 6.852 & -0.48 & 22.245 & 8.666 \\
\hline 0.4 & 16.196 & 6.761 & -0.47 & 22.321 & 8.716 \\
\hline 0.41 & 16.114 & 6.665 & -0.46 & 22.402 & 8.773 \\
\hline 0.42 & 16.039 & 6.574 & -0.45 & 22.477 & 8.823 \\
\hline 0.43 & 15.964 & 6.484 & -0.44 & 22.553 & 8.874 \\
\hline 0.44 & 15.895 & 6.401 & -0.43 & 22.628 & 8.925 \\
\hline 0.45 & 15.82 & 6.311 & -0.42 & 22.703 & 8.976 \\
\hline 0.46 & 15.744 & 6.222 & -0.41 & 22.779 & 9.027 \\
\hline 0.47 & 15.675 & 6.139 & -0.4 & 22.848 & 9.072 \\
\hline 0.48 & 15.606 & 6.056 & -0.39 & 22.923 & 9.124 \\
\hline 0.49 & 15.537 & 5.973 & -0.38 & 22.992 & 9.17 \\
\hline 0.5 & 15.468 & 5.89 & -0.37 & 23.067 & 9.222 \\
\hline 0.51 & 15.399 & 5.808 & -0.36 & 23.136 & 9.268 \\
\hline 0.52 & 15.33 & 5.725 & -0.35 & 23.205 & 9.314 \\
\hline 0.53 & 15.261 & 5.643 & -0.34 & 23.274 & 9.36 \\
\hline 0.54 & 15.192 & 5.561 & -0.33 & 23.337 & 9.4 \\
\hline 0.55 & 15.129 & 5.485 & -0.32 & 23.406 & 9.446 \\
\hline 0.56 & 15.067 & 5.41 & -0.31 & 23.469 & 9.487 \\
\hline 0.57 & 14.997 & 5.328 & -0.3 & 23.538 & 9.534 \\
\hline 0.58 & 14.935 & 5.253 & -0.29 & 23.601 & 9.575 \\
\hline 0.59 & 14.872 & 5.178 & -0.28 & 23.657 & 9.609 \\
\hline 0.6 & 14.809 & 5.103 & -0.27 & 23.72 & 9.65 \\
\hline 0.61 & 14.746 & 5.028 & -0.26 & 23.776 & 9.685 \\
\hline 0.62 & 14.684 & 4.953 & -0.25 & 23.833 & 9.72 \\
\hline 0.63 & 14.621 & 4.879 & -0.24 & 23.889 & 9.755 \\
\hline 0.64 & 14.558 & 4.804 & -0.23 & 23.946 & 9.791 \\
\hline 0.65 & 14.502 & 4.736 & -0.22 & 24.002 & 9.826 \\
\hline 0.66 & 14.439 & 4.662 & -0.21 & 24.052 & 9.856 \\
\hline 0.67 & 14.383 & 4.595 & -0.2 & 24.103 & 9.885 \\
\hline 0.68 & 14.32 & 4.521 & -0.19 & 24.147 & 9.909 \\
\hline 0.69 & 14.263 & 4.453 & -0.18 & 24.197 & 9.939 \\
\hline 0.7 & 14.207 & 4.386 & -0.17 & 24.241 & 9.962 \\
\hline 0.71 & 14.15 & 4.319 & -0.16 & 24.285 & 9.986 \\
\hline 0.72 & 14.094 & 4.251 & -0.15 & 24.322 & 10.004 \\
\hline 0.73 & 14.037 & 4.184 & -0.14 & 24.366 & 10.029 \\
\hline 0.74 & 13.981 & 4.118 & -0.13 & 24.404 & 10.047 \\
\hline
\end{tabular}




\begin{tabular}{|c|c|c|c|c|c|}
\hline 0.75 & 13.924 & 4.051 & -0.12 & 24.435 & 10.058 \\
\hline 0.76 & 13.868 & 3.984 & -0.11 & 24.473 & 10.077 \\
\hline 0.77 & 13.811 & 3.918 & -0.1 & 24.504 & 10.089 \\
\hline 0.78 & 13.755 & 3.851 & -0.09 & 24.529 & 10.095 \\
\hline 0.79 & 13.705 & 3.791 & -0.08 & 24.561 & 10.108 \\
\hline 0.8 & 13.648 & 3.725 & -0.07 & 24.58 & 10.108 \\
\hline 0.81 & 13.598 & 3.665 & -0.06 & 24.605 & 10.114 \\
\hline 0.82 & 13.542 & 3.599 & -0.05 & 24.623 & 10.114 \\
\hline 0.83 & 13.491 & 3.54 & -0.04 & 24.642 & 10.115 \\
\hline 0.84 & 13.435 & 3.474 & -0.03 & 24.661 & 10.116 \\
\hline 0.85 & 13.385 & 3.415 & -0.02 & 24.674 & 10.11 \\
\hline 0.86 & 13.335 & 3.356 & -0.01 & 24.68 & 10.098 \\
\hline 0.87 & 13.284 & 3.297 & 0 & 24.699 & 10.1 \\
\hline 0.88 & 13.234 & 3.238 & 0.01 & 24.68 & 10.073 \\
\hline 0.89 & 13.184 & 3.179 & 0.02 & 24.674 & 10.056 \\
\hline 0.9 & 13.134 & 3.12 & 0.03 & 24.661 & 10.034 \\
\hline 0.91 & 13.084 & 3.061 & 0.04 & 24.642 & 10.008 \\
\hline 0.92 & 13.033 & 3.003 & 0.05 & 24.623 & 9.982 \\
\hline 0.93 & 12.983 & 2.944 & 0.06 & 24.605 & 9.956 \\
\hline 0.94 & 12.933 & 2.886 & 0.07 & 24.58 & 9.926 \\
\hline 0.95 & 12.883 & 2.828 & 0.08 & 24.548 & 9.891 \\
\hline 0.96 & 12.839 & 2.776 & 0.09 & 24.523 & 9.861 \\
\hline 0.97 & 12.789 & 2.718 & 0.1 & 24.485 & 9.822 \\
\hline 0.98 & 12.738 & 2.66 & 0.11 & 24.454 & 9.788 \\
\hline 0.99 & 12.695 & 2.608 & 0.12 & 24.416 & 9.749 \\
\hline 1 & 12.644 & 2.55 & 0.13 & 24.372 & 9.706 \\
\hline 1.01 & 12.6 & 2.499 & 0.14 & 24.329 & 9.663 \\
\hline 1.02 & 12.55 & 2.441 & 0.15 & 24.285 & 9.62 \\
\hline 1.03 & 12.506 & 2.39 & 0.16 & 24.234 & 9.572 \\
\hline 1.04 & 12.462 & 2.339 & 0.17 & 24.184 & 9.525 \\
\hline 1.05 & 12.412 & 2.281 & 0.18 & 24.128 & 9.473 \\
\hline 1.06 & 12.368 & 2.23 & 0.19 & 24.071 & 9.422 \\
\hline 1.07 & 12.324 & 2.18 & 0.2 & 24.009 & 9.366 \\
\hline 1.08 & 12.28 & 2.129 & 0.21 & 23.946 & 9.31 \\
\hline 1.09 & 12.236 & 2.078 & 0.22 & 23.883 & 9.254 \\
\hline 1.1 & 12.186 & 2.021 & 0.23 & 23.814 & 9.194 \\
\hline 1.11 & 12.142 & 1.971 & 0.24 & 23.745 & 9.134 \\
\hline 1.12 & 12.098 & 1.92 & 0.25 & 23.67 & 9.069 \\
\hline 1.13 & 12.054 & 1.87 & 0.26 & 23.594 & 9.005 \\
\hline 1.14 & 12.011 & 1.82 & 0.27 & 23.519 & 8.94 \\
\hline 1.15 & 11.973 & 1.776 & 0.28 & 23.437 & 8.872 \\
\hline 1.16 & 11.929 & 1.725 & 0.29 & 23.356 & 8.803 \\
\hline 1.17 & 11.885 & 1.675 & 0.3 & 23.268 & 8.73 \\
\hline 1.18 & 11.841 & 1.625 & 0.31 & 23.18 & 8.657 \\
\hline
\end{tabular}




\begin{tabular}{|c|c|c|c|c|c|}
\hline 1.19 & 11.797 & 1.576 & 0.32 & 23.092 & 8.584 \\
\hline 1.2 & 11.76 & 1.532 & 0.33 & 22.998 & 8.507 \\
\hline 1.21 & 11.716 & 1.482 & 0.34 & 22.904 & 8.43 \\
\hline 1.22 & 11.672 & 1.433 & 0.35 & 22.81 & 8.353 \\
\hline 1.23 & 11.634 & 1.389 & 0.36 & 22.71 & 8.271 \\
\hline 1.24 & 11.59 & 1.34 & 0.37 & 22.609 & 8.19 \\
\hline 1.25 & 11.552 & 1.297 & 0.38 & 22.509 & 8.109 \\
\hline 1.26 & 11.509 & 1.247 & 0.39 & 22.408 & 8.028 \\
\hline 1.27 & 11.471 & 1.204 & 0.4 & 22.302 & 7.942 \\
\hline 1.28 & 11.427 & 1.155 & 0.41 & 22.195 & 7.857 \\
\hline 1.29 & 11.389 & 1.112 & 0.42 & 22.082 & 7.767 \\
\hline 1.3 & 11.352 & 1.069 & 0.43 & 21.969 & 7.677 \\
\hline 1.31 & 11.308 & 1.02 & 0.44 & 21.856 & 7.588 \\
\hline 1.32 & 11.27 & 0.978 & 0.45 & 21.743 & 7.498 \\
\hline 1.33 & 11.232 & 0.935 & 0.46 & 21.63 & 7.409 \\
\hline 1.34 & 11.188 & 0.886 & 0.47 & 21.511 & 7.315 \\
\hline 1.35 & 11.151 & 0.844 & 0.48 & 21.392 & 7.222 \\
\hline 1.36 & 11.113 & 0.801 & 0.49 & 21.273 & 7.128 \\
\hline 1.37 & 11.076 & 0.759 & 0.5 & 21.153 & 7.035 \\
\hline 1.38 & 11.038 & 0.716 & 0.51 & 21.028 & 6.937 \\
\hline 1.39 & 11 & 0.674 & 0.52 & 20.909 & 6.844 \\
\hline 1.4 & 10.963 & 0.632 & 0.53 & 20.783 & 6.746 \\
\hline 1.41 & 10.925 & 0.59 & 0.54 & 20.658 & 6.649 \\
\hline 1.42 & 10.887 & 0.548 & 0.55 & 20.532 & 6.551 \\
\hline 1.43 & 10.85 & 0.506 & 0.56 & 20.407 & 6.454 \\
\hline 1.44 & 10.812 & 0.464 & 0.57 & 20.275 & 6.353 \\
\hline 1.45 & 10.774 & 0.422 & 0.58 & 20.149 & 6.256 \\
\hline 1.46 & 10.737 & 0.38 & 0.59 & 20.018 & 6.154 \\
\hline 1.47 & 10.699 & 0.338 & 0.6 & 19.886 & 6.053 \\
\hline 1.48 & 10.668 & 0.303 & 0.61 & 19.754 & 5.952 \\
\hline 1.49 & 10.63 & 0.261 & 0.62 & 19.622 & 5.851 \\
\hline 1.5 & 10.592 & 0.22 & 0.63 & 19.49 & 5.75 \\
\hline 1.51 & 10.555 & 0.178 & 0.64 & 19.359 & 5.649 \\
\hline 1.52 & 10.523 & 0.143 & 0.65 & 19.227 & 5.549 \\
\hline 1.53 & 10.486 & 0.101 & 0.66 & 19.089 & 5.444 \\
\hline 1.54 & 10.454 & 0.066 & 0.67 & 18.957 & 5.343 \\
\hline 1.55 & 10.417 & 0.025 & 0.68 & 18.825 & 5.243 \\
\hline 1.56 & 10.379 & -0.017 & 0.69 & 18.687 & 5.138 \\
\hline 1.57 & 10.348 & -0.052 & 0.7 & 18.555 & 5.039 \\
\hline 1.58 & 10.31 & -0.093 & 0.71 & 18.417 & 4.934 \\
\hline 1.59 & 10.279 & -0.128 & 0.72 & 18.279 & 4.83 \\
\hline 1.6 & 10.241 & -0.169 & 0.73 & 18.148 & 4.73 \\
\hline 1.61 & 10.21 & -0.204 & 0.74 & 18.01 & 4.626 \\
\hline 1.62 & 10.178 & -0.238 & 0.75 & 17.878 & 4.527 \\
\hline
\end{tabular}




\begin{tabular}{|c|c|c|c|c|c|}
\hline 1.63 & 10.141 & -0.279 & 0.76 & 17.74 & 4.423 \\
\hline 1.64 & 10.109 & -0.314 & 0.77 & 17.602 & 4.319 \\
\hline 1.65 & 10.078 & -0.349 & 0.78 & 17.47 & 4.221 \\
\hline 1.66 & 10.04 & -0.389 & 0.79 & 17.332 & 4.117 \\
\hline 1.67 & 10.009 & -0.424 & 0.8 & 17.2 & 4.019 \\
\hline 1.68 & 9.977 & -0.459 & 0.81 & 17.062 & 3.915 \\
\hline 1.69 & 9.946 & -0.493 & 0.82 & 16.924 & 3.812 \\
\hline 1.7 & 9.908 & -0.534 & 0.83 & 16.792 & 3.714 \\
\hline 1.71 & 9.877 & -0.568 & 0.84 & 16.654 & 3.611 \\
\hline 1.72 & 9.846 & -0.602 & 0.85 & 16.522 & 3.513 \\
\hline 1.73 & 9.814 & -0.637 & 0.86 & 16.384 & 3.411 \\
\hline 1.74 & 9.783 & -0.671 & 0.87 & 16.252 & 3.313 \\
\hline 1.75 & 9.751 & -0.705 & 0.88 & 16.121 & 3.215 \\
\hline 1.76 & 9.72 & -0.739 & 0.89 & 15.983 & 3.113 \\
\hline 1.77 & 9.689 & -0.773 & 0.9 & 15.851 & 3.016 \\
\hline 1.78 & 9.657 & -0.807 & 0.91 & 15.719 & 2.918 \\
\hline 1.79 & 9.626 & -0.842 & 0.92 & 15.587 & 2.821 \\
\hline 1.8 & 9.595 & -0.876 & 0.93 & 15.456 & 2.724 \\
\hline 1.81 & 9.563 & -0.91 & 0.94 & 15.324 & 2.627 \\
\hline 1.82 & 9.532 & -0.944 & 0.95 & 15.192 & 2.53 \\
\hline 1.83 & 9.5 & -0.977 & 0.96 & 15.06 & 2.433 \\
\hline 1.84 & 9.475 & -1.005 & 0.97 & 14.928 & 2.337 \\
\hline 1.85 & 9.444 & -1.039 & 0.98 & 14.797 & 2.24 \\
\hline 1.86 & 9.413 & -1.073 & 0.99 & 14.665 & 2.144 \\
\hline 1.87 & 9.381 & -1.106 & 1 & 14.539 & 2.052 \\
\hline 1.88 & 9.35 & -1.14 & 1.01 & 14.408 & 1.956 \\
\hline 1.89 & 9.325 & -1.168 & 1.02 & 14.282 & 1.864 \\
\hline 1.9 & 9.293 & -1.201 & 1.03 & 14.15 & 1.768 \\
\hline 1.91 & 9.262 & -1.235 & 1.04 & 14.025 & 1.677 \\
\hline 1.92 & 9.237 & -1.262 & 1.05 & 13.899 & 1.586 \\
\hline 1.93 & 9.206 & -1.296 & 1.06 & 13.774 & 1.495 \\
\hline 1.94 & 9.174 & -1.329 & 1.07 & 13.648 & 1.404 \\
\hline 1.95 & 9.149 & -1.357 & 1.08 & 13.523 & 1.313 \\
\hline 1.96 & 9.118 & -1.39 & 1.09 & 13.397 & 1.223 \\
\hline 1.97 & 9.093 & -1.417 & 1.1 & 13.272 & 1.132 \\
\hline 1.98 & 9.061 & -1.451 & 1.11 & 13.146 & 1.041 \\
\hline 1.99 & 9.036 & -1.478 & 1.12 & 13.027 & 0.956 \\
\hline 2 & 9.005 & -1.511 & 1.13 & 12.902 & 0.865 \\
\hline 2.01 & 8.98 & -1.538 & 1.14 & 12.782 & 0.78 \\
\hline 2.02 & 8.955 & -1.565 & 1.15 & 12.657 & 0.69 \\
\hline 2.03 & 8.923 & -1.599 & 1.16 & 12.538 & 0.604 \\
\hline 2.04 & 8.898 & -1.626 & 1.17 & 12.418 & 0.519 \\
\hline 2.05 & 8.867 & -1.659 & 1.18 & 12.299 & 0.434 \\
\hline 2.06 & 8.842 & -1.686 & 1.19 & 12.18 & 0.349 \\
\hline
\end{tabular}




\begin{tabular}{|c|c|c|c|c|c|}
\hline 2.07 & 8.817 & -1.713 & 1.2 & 12.061 & 0.264 \\
\hline 2.08 & 8.785 & -1.746 & 1.21 & 11.948 & 0.184 \\
\hline 2.09 & 8.76 & -1.773 & 1.22 & 11.829 & 0.099 \\
\hline 2.1 & 8.735 & -1.8 & 1.23 & 11.716 & 0.019 \\
\hline 2.11 & 8.71 & -1.827 & 1.24 & 11.596 & -0.066 \\
\hline 2.12 & 8.685 & -1.853 & 1.25 & 11.483 & -0.146 \\
\hline 2.13 & 8.653 & -1.886 & 1.26 & 11.37 & -0.226 \\
\hline 2.14 & 8.628 & -1.913 & 1.27 & 11.258 & -0.305 \\
\hline 2.15 & 8.603 & -1.94 & 1.28 & 11.145 & -0.385 \\
\hline 2.16 & 8.578 & -1.967 & 1.29 & 11.032 & -0.464 \\
\hline 2.17 & 8.553 & -1.993 & 1.3 & 10.925 & -0.539 \\
\hline 2.18 & 8.528 & -2.02 & 1.31 & 10.812 & -0.619 \\
\hline \multirow[t]{32}{*}{2.19} & 8.503 & -2.047 & 1.32 & 10.705 & -0.693 \\
\hline & & & 1.33 & 10.592 & -0.772 \\
\hline & & & 1.34 & 10.486 & -0.847 \\
\hline & & & 1.35 & 10.379 & -0.921 \\
\hline & & & 1.36 & 10.272 & -0.996 \\
\hline & & & 1.37 & 10.166 & -1.07 \\
\hline & & & 1.38 & 10.065 & -1.139 \\
\hline & & & 1.39 & 9.959 & -1.213 \\
\hline & & & 1.4 & 9.858 & -1.283 \\
\hline & & & 1.41 & 9.758 & -1.352 \\
\hline & & & 1.42 & 9.657 & -1.421 \\
\hline & & & 1.43 & 9.557 & -1.49 \\
\hline & & & 1.44 & 9.457 & -1.559 \\
\hline & & & 1.45 & 9.356 & -1.628 \\
\hline & & & 1.46 & 9.262 & -1.692 \\
\hline & & & 1.47 & 9.162 & -1.761 \\
\hline & & & 1.48 & 9.068 & -1.825 \\
\hline & & & 1.49 & 8.973 & -1.889 \\
\hline & & & 1.5 & 8.879 & -1.953 \\
\hline & & & 1.51 & 8.785 & -2.017 \\
\hline & & & 1.52 & 8.691 & -2.081 \\
\hline & & & 1.53 & 8.603 & -2.14 \\
\hline & & & 1.54 & 8.515 & -2.199 \\
\hline & & & 1.55 & 8.421 & -2.263 \\
\hline & & & 1.56 & 8.333 & -2.322 \\
\hline & & & 1.57 & 8.252 & -2.376 \\
\hline & & & 1.58 & 8.164 & -2.434 \\
\hline & & & 1.59 & 8.076 & -2.493 \\
\hline & & & 1.6 & 7.994 & -2.547 \\
\hline & & & 1.61 & 7.913 & -2.601 \\
\hline & & & 1.62 & 7.831 & -2.655 \\
\hline & & & 1.63 & 7.75 & -2.709 \\
\hline
\end{tabular}




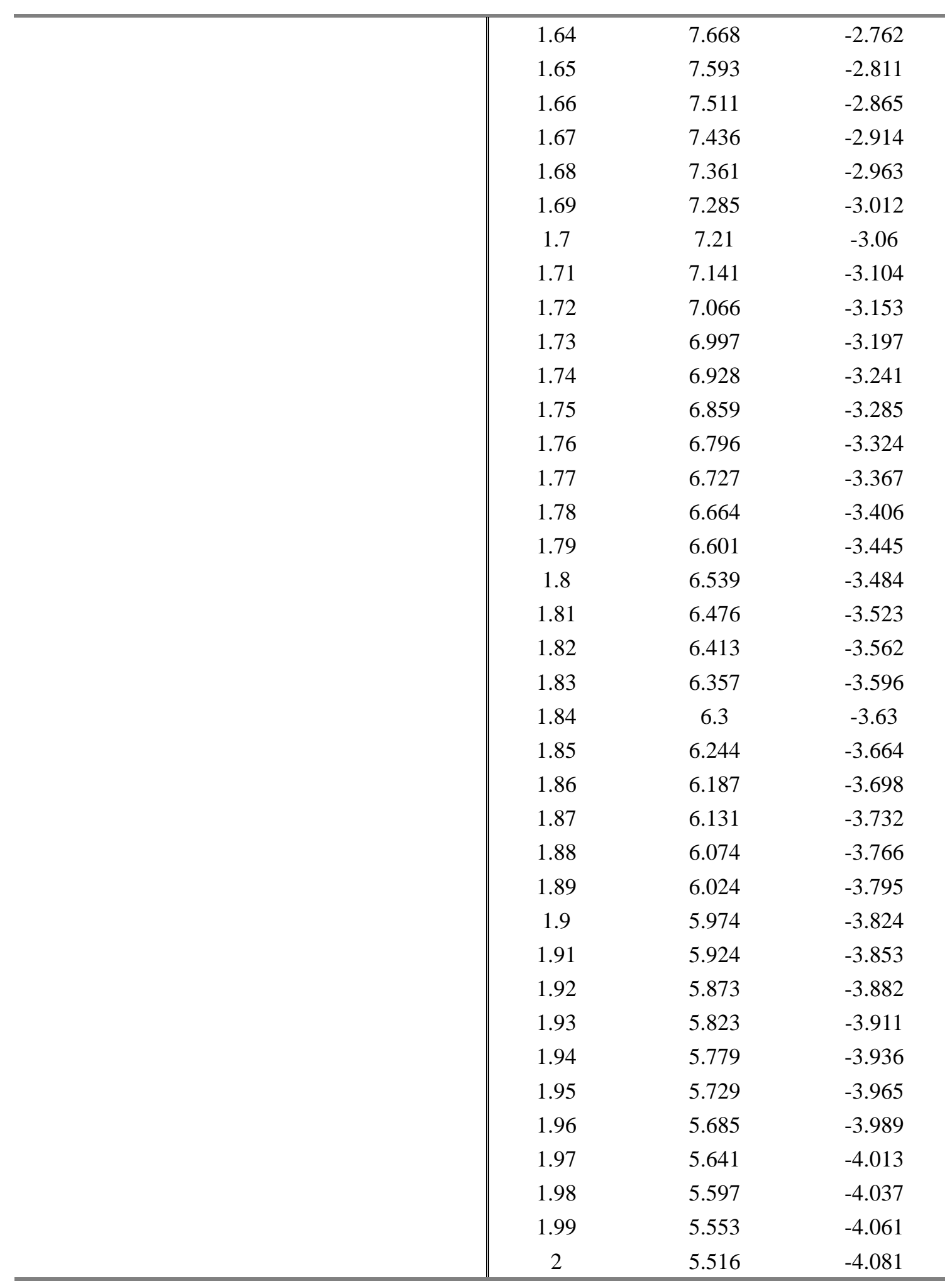

